\title{
Numerical Investigation of Sloshing Under Roll Excitation at Shallow Liquid Depths and the Effect of Baffles
}

\author{
Arun Kamath $^{1} \cdot$ Erlend Liavåg Grotle ${ }^{2} \cdot$ Hans Bihs $^{1}$ \\ Received: 7 October 2020 / Accepted: 24 December 2020 / Published online: 2 June 2021 \\ (C) The Author(s) 2021
}

\begin{abstract}
Sloshing is relevant in several applications like ship tanks, space and automotive industry and seiching in harbours. Due to the relationship between ship and sloshing motions and possibility of structural damage, it is important to represent this phenomenon accurately. This paper investigates sloshing at shallow liquid depths in a rectangular container using experiments and RANS simulations. Free and forced sloshing, with and without baffles, are studied at frequencies chosen specifically in proximity to the first mode natural frequency. The numerically calculated free surface elevation is in close agreement with observations from experiments. The upper limit of the resonance zone, sloshing under different filling depths and roll amplitudes and sloshing with one, two and four baffles are also investigated. The results show that the extent of the resonance zone is reduced for higher filling depth and roll amplitude. It is also found that the inclusion of baffles moves the frequency at which the maximum free surface elevation occurs, away from the fundamental frequency. Finally, a submerged baffle is found to dissipate more energy compared to a surface piercing baffle and that the effect of several submerged baffles is similar to that of a single submerged baffle.
\end{abstract}

Keywords Sloshing $\cdot$ Baffles $\cdot$ Resonance $\cdot$ Numerical modelling $\cdot$ REEF3D

\section{Introduction}

Sloshing can be characterised as the motion of a liquid in contact with a much less dense fluid separated by an interface in a container or vessel. There are several applications where sloshing should be considered. The essential parameters to be evaluated in the analysis of sloshing are the hydrodynamic pressure distribution, forces, moments and natural frequencies. Extensive works on analytical approaches to

\section{Article Highlights}

- Open-source CFD model REEF3D was employed to study sloshing with and without baffles.

- The resonance region under sloshing for different roll amplitudes and filling ratios was investigated.

- Sloshing with a single tall baffle and multiple short baffles was simulated.

- Tank configured with multiple small baffles reduce the sloshing amplitudes better than a single tall baffle.

Arun Kamath

arun.kamath@ntnu.no

1 Department of Civil and Environmental Engineering, Norwegian University of Science and Technology, 7491 Trondheim, Norway

2 Kongsberg Maritime CM AS, 6065 Ulsteinvik, Norway study sloshing are found in Ibrahim (2005) and Faltinsen and Timokha (2009). While the former focuses on space applications, the latter presents sloshing within marine engineering. In marine applications, tanks have different applications, but in general, all marine vehicles have a tank of some kind installed on board. Examples are roll-stabiliser tanks or cargo tanks carrying different type of liquids. With a roll-stabilising system, the interaction between the ship motion and the tank sloshing is of interest, as the main purpose of these tanks is to maintain stability of the ship. Sloshing impact forces can cause severe damages on the tank structure. A major part of the research on sloshing is therefore devoted to finding the sloshing excited forces and moments.

The motion of the liquid has an infinite number of natural frequencies, but the lowest few modes are most likely to be excited by the motion of a vessel or vehicle (Ibrahim et al. 2001). The first mode natural frequency is of particular interest because excitation of the first mode results in the most severe sloshing. In order to choose the relevant sloshing regimes, it is helpful to calculate the natural frequencies. In this way, the motion parameters and filling can be chosen specifically.

The natural frequencies and modes depend on the geometry of the tank. In this study, a rectangular tank is used, 
because it has a simple geometry, with vertical side walls, and all walls are perpendicular to each other. The natural frequencies and corresponding modes can be found by solving the linearised free surface boundary problem with zero tank excitation (Faltinsen and Timokha 2009).

An important parameter in characterising sloshing is the relative liquid depth $h / L$, where $h$ is the depth of filling and $L$ is the length of the sloshing tank relationship. The hyperbolic tangent term in the equation for sloshing behaves differently for small and large values of $h / L$. At shallow water conditions, $\tanh (\pi i h / L) \approx \pi i h / L$, which is practical when $h / L \leq$ 0.05-0.1 (Faltinsen and Timokha 2009). Hydraulic jumps may be formed around resonance in the shallow liquid case. Verhagen and Van Wijngaarden (1965) developed a steadystate theory under these conditions based on non-linear shallow liquid potential flow theory. They conducted experiments with a forced harmonic roll motion and compared it with analysis. The criteria for the hydraulic jump to occur at roll excitation predict hydraulic jumps when $f / f_{1,0}=1$ for any forcing amplitude. Faltinsen and Timokha (2009) developed further the theory for forced sway. Tests performed by Lugni showed that the hydraulic jump did not necessarily occur as predicted by theory, because it is sensitive to the actual depths (Faltinsen and Timokha 2009).

The analytical methods in sloshing use the modal approach to the velocity potential and the free surface. This means that the free surface motion is decomposed into several Fouriertype modes. These methods are considered to be exact solutions of the sloshing problem but based on the assumption of an inviscid fluid. In Faltinsen et al. (2000), the theoretical background for the non-linear modal modelling of potential flow in sloshing is given. Overturning waves are excluded in this model, and it is not valid for shallow water depths. Energy dissipation is an additional factor that must be accounted for in these methods, which is difficult because it consists of different and unknown factors, especially the energy dissipation due to turbulence. This process was studied for closed basins in Keulegan (1959) and Miles (1967). The calculated dissipation was almost always found to be underpredicted compared to experiments. At shallow liquid depths, the sloshing is violent and highly non-linear, and an infinite number of modes are needed to describe the free surface motion. The dissipation of energy is also significant in resonant sloshing at shallow depths. Faltinsen and Timokha (2002) revised the modal system theory describing non-linear sloshing to match intermediate and shallow fluid depths. The results are accurate when dissipation is taken into account but are valid only for limited fluid depths. Energy dissipation due to run-up along the vertical walls and wave breaking is difficult to account for in such models. Studies performed by Armenio and La Rocca (1996) investigated the variation of the wave elevation with roll amplitude for different depths and found that the resonance zone is enlarged depending on the roll amplitude and filling. The studies restricted the roll amplitude varied between 1.0 and $4.5^{\circ}$ with a low filling depth and excitation frequencies between 0.48 and $0.8 \mathrm{~Hz}$. Antuono et al. (2012) developed a two-dimensional modal method for sloshing in rectangular tanks with shallow liquid depths. They compared the model with experimental data and SPH simulations. Sloshing with roll motion was compared only to $\mathrm{SPH}$, due to the lack of experimental data with non-breaking wave regimes. They concluded that the methods proved to be robust and accurate and provide a good description of sloshing motions when the water depth is shallow, but without breaking waves.

To investigate the detailed flow conditions in sloshing, both with and without internal structures, CFD methods provide promising capabilities. Viscous damping can be modelled accurately, and wave breaking regimes can also be accounted for. In Jung et al. (2012), the effect of the baffle height is investigated numerically using RANS with the $k-\varepsilon$ turbulence model. The results are compared with experimental values of impact pressure at the side walls, and the agreement with experimental data are satisfactorily. Wu et al. (2012) performed extensive numerical analysis of the influence of baffles on the first mode natural frequency in twodimensional rectangular tanks. They solved the RANS equations and employed a fictitious cell method to resolve vortices generated around the baffle tips. The free surface elevation results from simulations were compared to experimental data and showed acceptable agreement. Zhao and Chen (2015) performed numerical analysis of sloshing in a three-dimensional LNG membrane tank investigating impact pressures on the side walls. They implemented a new coupled level-set and volume-of-fluid method to handle the free surface motion. A finite-analytical Navier-Stokes (FANS) was employed to solve momentum. They concluded that the new scheme maintained stable mass conservation within each of the cells and that the method demonstrated capabilities in the accurate prediction of LNG sloshing impacts. Lu et al. (2015) studied the effect of baffles in a 2D rectangular tank. They compared the results from a viscous finite-element solver (FEM) with results from potential flow theory. In the case with a clean tank, they found that analytical potential flow solutions over-predicted the free surface amplitude in the longer time series, while with baffles the discrepancies are seen due to strong energy losses. The difference is attributed to the lack of viscous effects in the potential flow solutions.

In order to explore numerical modelling to simulate sloshing, the Reynolds-averaged Navier-Stokes (RANS) equation-based solver REEF3D is used in this study. The model has been extensively used for wave hydrodynamics problems (Ong et al. 2017), violent water impact (Kamath et al. 2017) and sediment transport problems (Ahmad et al. 2018), sloshing at shallow water depths (Grotle et al. 2017) and non-linear sloshing (Grotle et al. 2018). The $k-\omega$ model 
(Wilcox 1994) is used for turbulence modelling. Forced sloshing within the proximity of the first mode resonance and free sloshing is simulated and compared to experiments performed at the lab facility at NTNU in Ålesund. In this paper, free sloshing is carried out to identify the natural frequencies followed by sloshing at different excitation frequencies close to the first mode of sloshing. The experimental observations are used to validate the numerical model. It is found that the simulations predict the free surface elevation at steady-state sloshing accurately, also when the excitation frequency is close to the first fundamental frequency. At greater frequencies, with activation of higher mode overlapping waves, some discrepancy is observed. The transition sloshing regime in the upper resonance zone, the effect of higher tank filling, larger roll amplitudes and the effect of baffles on the sloshing regime are then investigated using the simulations.

\section{Description of the Numerical Model}

The open-source hydrodynamic model REEF3D (Bihs et al. 2016; Bihs and Kamath 2017) is used in this study. The flow is assumed to be incompressible with constant density and molecular viscosity in the gas and liquid phase, neglecting compressibility and thermal effects. The governing equations are solved numerically using a finite difference method. A single set of equations describes the entire flow field. The immersed boundary method is used to define the boundaries of complex objects in the domain.

\subsection{RANS Equations}

We assume incompressible flow and a Newtonian fluid. Splitting into averaging and fluctuating terms, and combining the continuity equation, gives the simplified RANS equation (Wilcox 1994):

$$
\begin{gathered}
\frac{\partial u_{i}}{\partial x_{i}}=0 \\
\frac{\partial u_{i}}{\partial t}+u_{j} \frac{\partial u_{i}}{\partial x_{j}}=-\frac{1}{\rho} \frac{\partial p}{\partial x_{i}}+\frac{\partial}{\partial x_{j}}\left[\left(\nu+\nu_{t}\right)\left(\frac{\partial u_{i}}{\partial x_{j}}+\frac{\partial u_{j}}{\partial x_{i}}\right)\right]+f_{i}
\end{gathered}
$$

where $u$ is the velocity, $t$ is time, $p$ is the pressure, $\nu$ is the kinematic viscosity and $\nu_{t}$ is the eddy viscosity. The term $f_{i}$ represents the body forces. As a tank-fixed coordinate system is used for simulating the sloshing, source terms in addition to gravity must be accounted for to represent the equations in a non-inertial global system, assuming planar rotational motion. The Poisson pressure equation is solved using a fully parallelised Jacobi-preconditioned BiCGStab algorithm (Van der Vorst 1992). Convective terms in the RANS equation are discretised using the conservative fifth-order WENO scheme
(Jiang and Shu 1996). The Hamilton-Jacobi formulation of the WENO scheme (Jiang and Peng 2000) is used to discretise the convective terms in the level set equation and in the $k$ and $\omega$ equations. The weighted essentially non-oscillatory scheme (WENO) uses a weight parameter and combines three essentially non-oscillatory (ENO) stencils. All temporal discretisation is done with the third-order TVD Runge-Kutta scheme (Harten 1983), except for the turbulence model equations. Adaptive time stepping ensures that the CFL criteria are fulfilled. The numerical grid is uniform and orthogonal. A local directional ghost cell immersed boundary method (Berthelsen and Faltinsen 2008) is implemented to account for solid boundaries, extended to three dimensions to handle multi-directional ghost cells.

\subsection{Turbulence}

Modelling turbulence in sloshing, or general free surface flow with large density ratios, is a complex task. The two equations $k-\omega$ turbulence model (Wilcox 1994) is used to close the set of RANS equations. The two equations govern the kinetic turbulence energy $k$ and the specific dissipation rate of turbulence energy $\omega$ and can be written as:

$$
\begin{gathered}
\frac{\partial k}{\partial t}+u_{j} \frac{\partial k}{\partial x_{j}}=\frac{\partial}{\partial x_{j}}\left[\left(\nu+\frac{\nu_{t}}{\sigma_{k}}\right) \frac{\partial k}{\partial x_{j}}\right]+P_{k}-\beta_{k} k \omega \\
\frac{\partial \omega}{\partial t}+u_{j} \frac{\partial \omega}{\partial x_{j}}=\frac{\partial}{\partial x_{j}}\left[\left(\nu+\frac{\nu_{t}}{\sigma_{\omega}}\right) \frac{\partial \omega}{\partial x_{j}}\right]+\frac{\omega}{k} \alpha P_{k}-\beta \omega^{2}
\end{gathered}
$$

$P_{k}$ is the turbulence energy production term, $\sigma_{k}$ and $\sigma_{\omega}$ are standard coefficients in the model, both with values of 2 , and $\beta_{k}, \beta$ and $\alpha$ are empirical constants, with values 9/100, 3/40 and $5 / 9$, respectively. Wall laws for velocity and turbulence energy are used, and no additional grid refinement is necessary at the wall boundaries. The RANS model overproduces the turbulence energy in highly strained flows. This gives unrealistically large values for the eddy viscosity. Menter (1994) noted that the stress intensity ratio scales with the ratio of turbulence production to dissipation. Typical stress intensity ratios can be found from experiments in certain type of flows. In order to avoid overproduction of turbulence in highly strained flow outside the boundary layer, the turbulent eddy viscosity, $\nu_{t}$, can be bounded through the limiting formulation (Durbin 2009):

$\nu_{t}=\min \left(\frac{k}{\omega}, \sqrt{\frac{2}{3}} \frac{k}{|S|}\right)$

where $S$ is the square root of rate of strain magnitude.

At the free surface, the turbulent length scales are reduced due to the free surface exerting similar effects as on wall boundaries, where a shear layer is formed due to the forces near the surface. The normal fluctuations are damped out, with 
an amplification of the other components. A boundary condition is proposed to limit the length scale near the free surface based on the observations by Naot and Rodi (1982):

$\omega_{s}=\frac{C_{\mu}^{-1 / 4}}{\kappa} k^{1 / 2} \cdot \frac{1}{y^{\prime}}$

where $C_{\mu}=0.07$ and $\kappa=0.4$, and $y^{\prime}$ is the virtual origin of the length scale of the turbulence. In Hossain and Rodi (1980), this was determined to be 0.07 times the mean water depth. To activate this boundary condition at the interface of thickness $\varepsilon=1.6 \mathrm{~d} x$, the expression is multiplied by the Dirac delta function:

$\delta(\phi)= \begin{cases}\frac{1}{2 \epsilon}\left[1+\cos \left(\frac{\pi \phi}{\epsilon}\right)\right] & , \text { if }|\phi|<\epsilon ; \\ 0 & \text { otherwise }\end{cases}$

where $\phi$ is the level set function. It should be noted that in Eq. (2), the eddy viscosity is limited in general. The free surface boundary condition of $\omega$ in Eq. (3) increases the dissipation and therefore reduces the eddy viscosity, but only at the free surface. Any reduction of $y^{\prime}$ increases $\omega$ and therefore increases the turbulent energy dissipation from the eddy viscosity.

\subsection{Interface Coupling}

The interface between liquid and gas represents a discontinuity in the field. It is therefore necessary to know the location of it at all times. To capture the interface, the level set technique is used, first presented by Osher and Sethian (1988). The location of the surface is represented by the zero level of a signed distance function. The following properties are defined:

$\phi(x, t)=\left\{\begin{array}{lc}>0, & \text { if } x \in \text { phase } 1 ; \\ =0, & \text { if } x \in \Gamma ; \\ <0, & \text { if } x \in \text { phase 2; }\end{array}\right.$

where $\Gamma$ is the free surface. In order to move the interface inside a velocity field, the level set function, $\phi$, is convected using the equation:

$\frac{\partial \phi}{\partial t}+\bar{u}_{j} \frac{\partial \phi}{\partial x_{j}}=0$

When the level set function is moved, it will not remain a signed distance function. For this to be fulfilled, it must satisfy the Eikonal equation also in order to ensure mass conservation. Reinitialisation at each time step is done using a partial differential equation- based method (Sussman et al. 1994; Peng et al. 1999). For the treatment of the abrupt change of fluid properties at the interface, the values are smoothed across the free surface over an interface thickness of $2.1 \mathrm{~d} x$ with a Heaviside function, $\mathrm{H}(\phi)$, as follows:

$$
\begin{aligned}
& \rho(\phi)=\rho_{1} H(\phi)+\rho_{2}[1-H(\phi)] \\
& \mu(\phi)=\mu_{1} H(\phi)+\mu_{2}[1-H(\phi)]
\end{aligned}
$$

\section{Experimental and Numerical Investigation}

The experiments were carried out at NTNU in Ålesund. A solid rotational platform is used to excite sloshing. A rectangular tank with shallow water depth is used. The tank is $1 \mathrm{~m}$ long and $0.15 \mathrm{~m}$ high. The height of the tank was found to be limited in some of the test cases, resulting in roof impact of the sloshing liquid. The effect of the roof impact is then studied in an experiment with an increased tank height of $0.3 \mathrm{~m}$. The water depth in all tests without a baffle is $h=0.06 \mathrm{~m}$, giving a water depth-to-tank length ratio $h / L=0.06$. The roll excitation amplitude is constant and equal to $3.0^{\circ}$ in all the tests. The roll axis is at the intersection of the bottom and the transverse centre plane of the tank, creating liquid motions in the longitudinal direction. A video of the sloshing is recorded. The distance from the tank top to the free surface is measured using

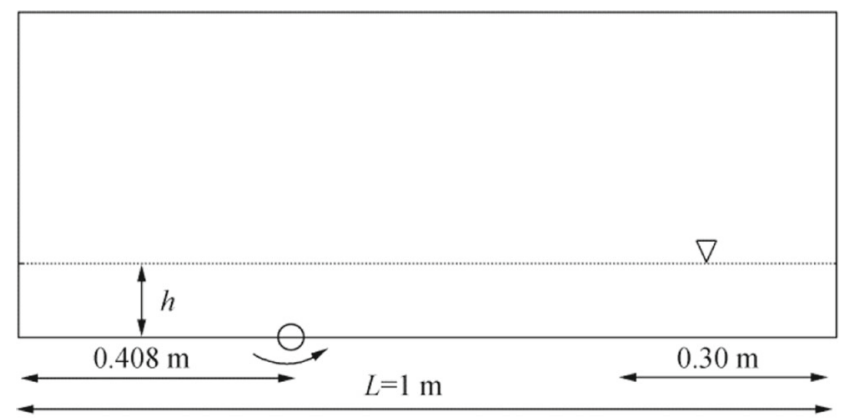

(a) Studies without baffles

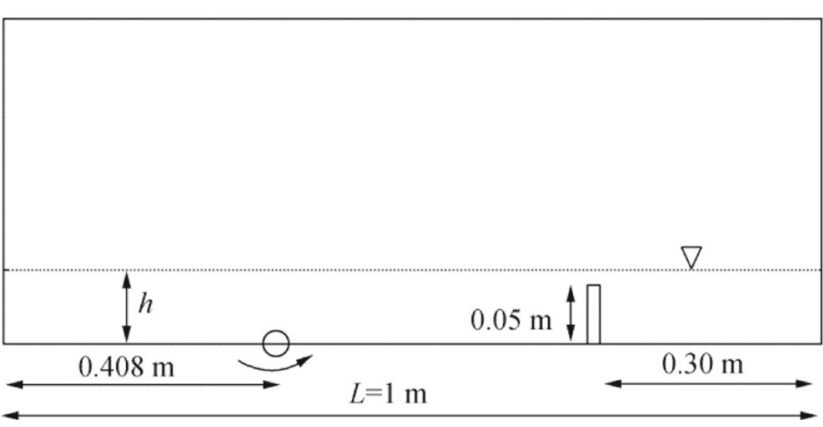

(b) Studies with baffles

Figure 1 Illustration of the sloshing tanks used in the experimental and numerical investigations 
an ultrasonic sensor, type Sick UM12, which is placed at a distance of $x=0.408 \mathrm{~m}$ from the left wall. The test setup for the experiments without a baffle is illustrated in Figure 1a. The resolution of the sensor is $0.069 \mathrm{~mm}$. The accuracy is $\pm 1 \%$ with a repeatability of $\pm 0.15 \%$ based on the current measurement value. A single baffle configuration is investigated with the same setup but with an increased mean water level such that $h / L=0.07$. The height of the baffle is $0.05 \mathrm{~m}$, providing a freeboard of $0.02 \mathrm{~m}$ in the sloshing tank as shown in Figure $1 b$. This configuration is referred to as the tall baffle as it pierces the free surface during sloshing motion.

The frequency of the motion is changed in the different tests, and theoretical natural frequencies are calculated. The tank breadth is small relative to the length, and so the natural frequencies corresponding to modes $j>0$ are higher, and therefore in most cases, 3D effects are negligible. The frequencies chosen are given in Table 1 along with the ratios of the harmonics of the excitation frequencies and the corresponding harmonics of the natural frequencies. The chosen excitation frequencies are in proximity to the first fundamental frequency, or higher. With an excitation frequency above the fundamental frequency, several waves are formed that overlap the primary wave. The sloshing regime close to the upper limit of the resonance zone is investigated more thoroughly in later sections by performing numerical simulations with several more frequencies. In addition, free sloshing is investigated in case 5 by stopping the platform after half a cycle. Generally it is found that after the stop, there is a transition period where no particular frequency is found. The reason for these investigations is that with free sloshing, the natural frequencies appear, which makes it possible to identify them with numerical simulations.

Numerical simulations are carried out to replicate all the experimental investigations listed above to validate the model. The study is then extended to investigate the transition region for sloshing, effect of roll amplitude (N0-N28), forced sloshing with a single tall baffle (B0-B7), forced sloshing with one (O0-O7), two (T0-T7) and four short baffles (F0F7). The baffle height in these cases with the short baffles is $0.015 \mathrm{~m}$, which corresponds to a baffle height to mean water depth ratio of 0.25 and does not pierce the free surface during the sloshing motion. In the simulations with a single small

Table 1 Excitation frequencies tested in experiments

\begin{tabular}{lllll}
\hline Case & $f(\mathrm{~Hz})$ & $f / f_{1,0}$ & $2 f / f_{2,0}$ & $3 f / f_{3,0}$ \\
\hline 1 & 0.30 & 0.79 & 0.80 & 0.82 \\
2 & 0.38 & 1.00 & 1.01 & 1.03 \\
3 & 0.53 & 1.40 & 1.43 & 1.60 \\
4 & 0.55 & 1.44 & 1.47 & 1.45 \\
5 & 0.17 & 0.44 & \multicolumn{3}{c}{ Single baffle } \\
6 & 0.23 & \multicolumn{3}{|l}{ Single baffle } \\
7 & 0.30 & \multicolumn{3}{c}{} \\
\hline
\end{tabular}

baffle $(\mathrm{O} 0-\mathrm{O} 7)$, the baffle is placed $0.30 \mathrm{~m}$ from one end of the tank, the same as that in simulations with a single baffle (B0-B7) and the experiments (cases 6 and 7). In the case with two baffles (T0-T7), the baffles are equally distributed with a distance of $0.33 \mathrm{~m}$. The tank layout with four baffles (F0-F7) is shown in Figure 2, where the four baffles are equally distributed with a distance of $0.2 \mathrm{~m}$ between two neighbouring baffles. Free sloshing is simulated for all the numerical simulations as well to ascertain the natural frequencies in the slightly modified tank configuration. An overview of the simulations carried out is provided in Tables 2, 3 and 4.

\section{Results and Discussion}

\subsection{Grid Convergence Study}

Three different cell sizes are compared for simulation N5, which corresponds to experimental case 3 . The grid is orthogonal and uniform. The resulting free surface elevation in the simulations is shown in Figure $3 \mathrm{a}$ along with the experimental observations. The simulations with grid $\mathrm{d} x=0.003 \mathrm{~m}$ provide similar results as $\mathrm{d} x=0.0025$ $\mathrm{m}$ while being significantly more computationally efficient, and therefore it is chosen for further studies. A grid sensitivity study is also carried out for a simulation with a single baffle B1, which corresponds to experimental case 6 . The free surface elevation in the experiments and the simulations at a distance of $0.408 \mathrm{~m}$ from the left wall are shown in Figure $3 \mathrm{~b}$. As in the grid sensitivity study in the simulations without baffles, it is seen that a grid size of $\mathrm{d} x=0.003 \mathrm{~m}$ is sufficient to represent the sloshing process reasonably well.

\subsection{Free Sloshing}

Free sloshing is investigated in the experimental case 5 by turning off the motion of the platform after half a cycle. This case evaluates the ability of the model in predicting wave speed and shape (Armenio and La Rocca 1996) and the appearance of higher natural modes. This is an efficient way to compare natural frequencies with theoretical values. The resulting free surface elevation is presented in Figure $4 \mathrm{a}$ for $100 \mathrm{~s}$ along with the corresponding simulated results from N0. Some discrepancy is seen in the free surface amplitudes, but in general, the natural frequencies are accurately predicted by the simulations. Since the free surface elevation is measured in the middle of the tank, a travelling wave in first mode resonance is

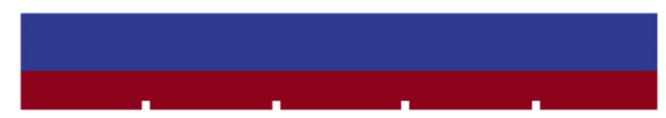

Figure 2 Numerical sloshing tank with four short baffles 
Table 2 Numerical investigation with no baffles

\begin{tabular}{lllll}
\hline Case & Roll $\left(^{\circ}\right)$ & $h / L$ & $f(\mathrm{~Hz})$ & $f / f_{1,0}$ \\
\hline N0/N10/N20 (free sloshing) & $3 / 6 / 1.7$ & 0.06 & 0.30 & 0.79 \\
N1/N11/N21 & $3 / 6 / 1.7$ & & 0.23 & 0.61 \\
N2/N12/N22 & $3 / 6 / 1.7$ & & 0.30 & 0.79 \\
N3/N13/N23 & $3 / 6 / 1.7$ & & 0.38 & 1.00 \\
N4/N14/N24 & $3 / 6 / 1.7$ & & 0.50 & 1.32 \\
N5/N15/N25 & $3 / 6 / 1.7$ & & 0.53 & 1.40 \\
N6/N16/N26 & $3 / 6 / 1.7$ & & 0.55 & 1.44 \\
N7/N17/N27 & $3 / 6 / 1.7$ & & 0.60 & 1.58 \\
N8/N18/N28 & $3 / 6 / 1.7$ & & 0.65 & 1.71 \\
H1 & 3 & 0.10 & 0.40 & 0.821 \\
H2 & & & 0.49 & 1.00 \\
H3 & & & 0.60 & 1.231 \\
H4 & & & 0.63 & 1.293 \\
H5 & & & 0.65 & 1.334 \\
H6 & & & 0.67 & 1.375 \\
\hline
\end{tabular}

captured twice - once when the wave travels from right to left and for a second time as it travels left to right after reflecting from the left wall. Therefore the signal is registered close to the second harmonic.

The spectrogram plot in Figure $4 \mathrm{~b}$ shows short-time Fourier transform of the experimental data. A time window of $12 \mathrm{~s}$ is used, which corresponds to 2 periods of the initial excitation. The overlap is $50 \%$. The figure shows that no distinct frequency dominates before approximately $15 \mathrm{~s}$. Two major peaks can be identified in the period $15-50 \mathrm{~s}$, around $0.75 \mathrm{~Hz}$ and at 1.10 , indicated by signals in the range of about $-30 \mathrm{~dB}$ and higher. An FFT analysis is performed after $25 \mathrm{~s}$ to find the natural frequencies. The FFT of both experiments and RANS is shown in Figure 5a. The peaks are close to the natural frequencies predicted by theory. It is seen that the RANS simulations predict slightly higher natural frequencies than the experiments. Further, the free sloshing is simulated with a single baffle (B0) and small baffles (S0, T0, F0) to investigate changes to the sloshing regime due to change in

Table 3 Numerical investigation with a single tall baffle
Table 4 Numerical investigation with short baffles

\begin{tabular}{lcc}
\hline Case & $f(\mathrm{~Hz})$ & $f / f_{1,0}$ \\
\hline O0/T0/F0 (free sloshing) & 0.30 & 0.79 \\
O1/T1/F1 & 0.23 & 0.61 \\
O2/T2/F2 & 0.30 & 0.79 \\
O3/T3/F3 & 0.38 & 1.00 \\
O4/T4/F4 & 0.50 & 1.32 \\
O5/T5/F5 & 0.55 & 1.44 \\
O6/T6/F6 & 0.60 & 1.58 \\
O7/T7/F7 & 0.65 & 1.71 \\
\hline
\end{tabular}

the structural elements in the sloshing tank. The FFT of the calculated time series for these four cases with baffles are presented in Figure 5b, and the natural frequency for all the cases with the baffles is seen to be $f_{0}=0.38 \mathrm{~Hz}$.

\subsection{Forced Sloshing Without Baffles}

In this section, the forced sloshing investigated through experiments and the validation of the numerical model to represent these cases is presented. In case 1, there is a single wave travelling back and forth with the forcing frequency. The wave rolls up at the end wall, and a spilling breaker is formed

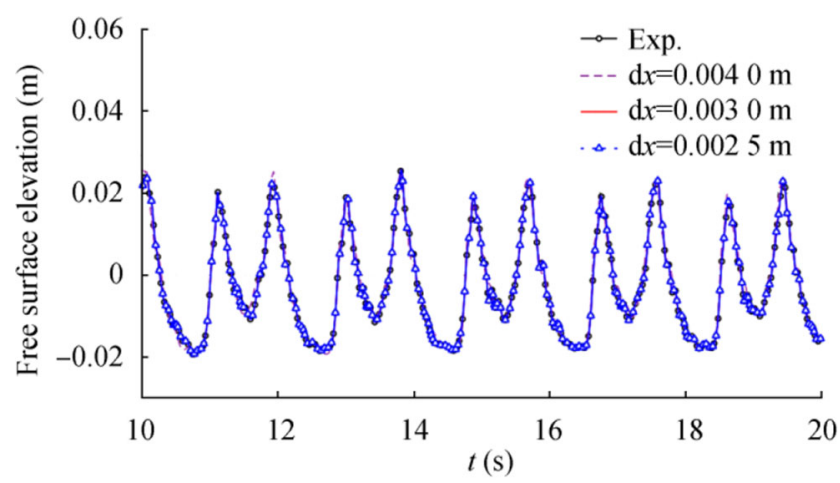

(a) Case N5 with $f=0.532 \mathrm{~Hz}$

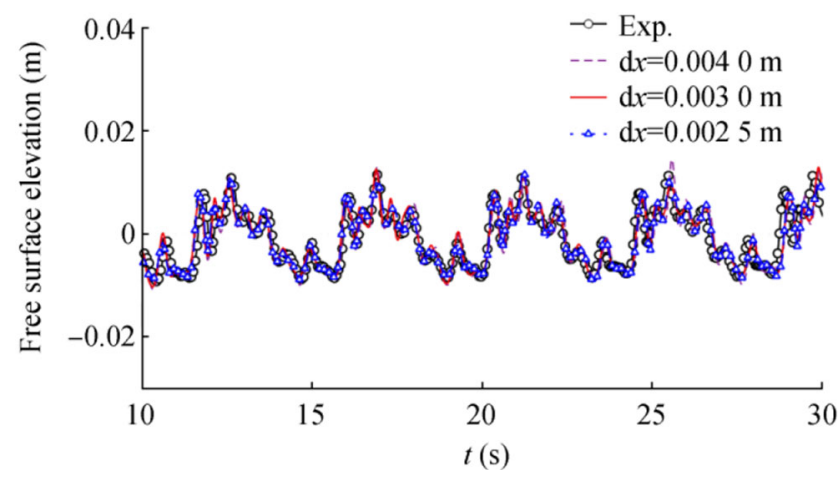

(b) Case B1 with $f=0.23 \mathrm{~Hz}$

Figure 3 Grid sensitivity in a simulation without baffles (N5) and a single baffle (B1) 


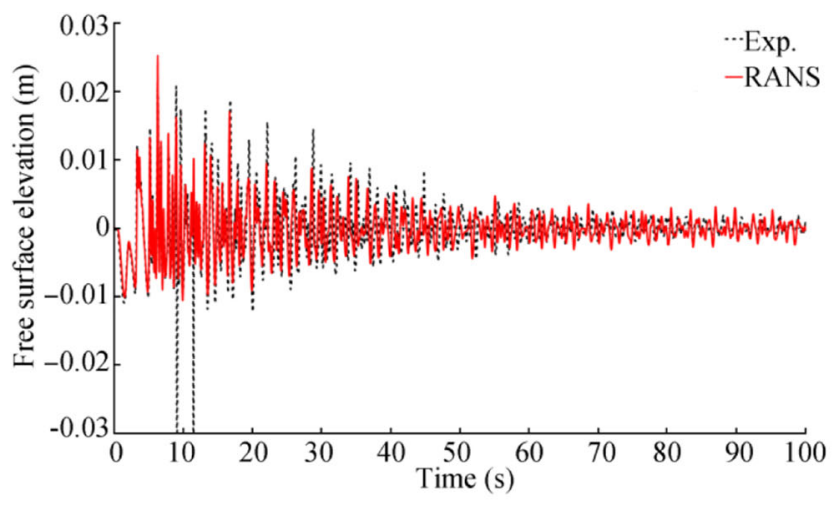

(a) Experiments (case 5) and numerical model (N0)

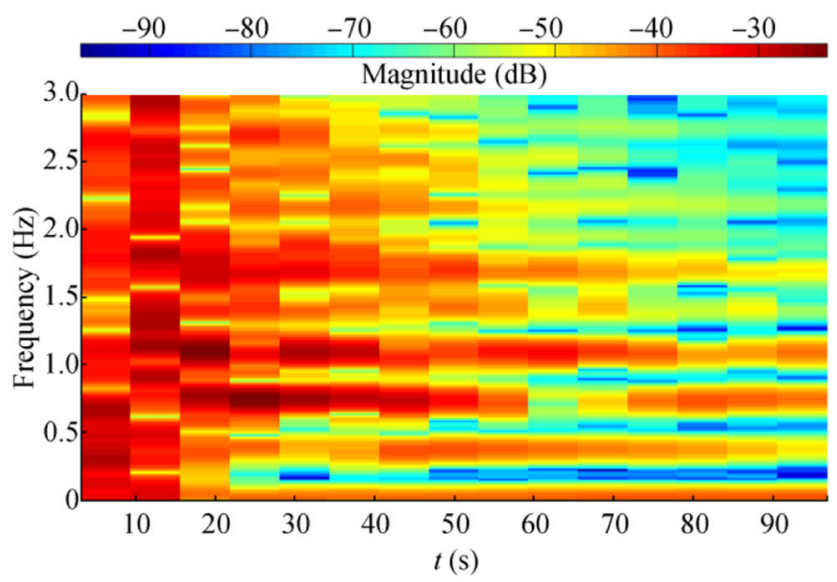

(b) Spectrogram showing dominant frequencies in the experiments

Figure 4 Free surface elevation and dominant frequencies under free sloshing without baffles

closely after the roll-up, as shown in Figure 6a. Case 2 is more violent, and a jet is formed when the spilling wave hits the wall. The jet hits the roof, the wave breaks and water reflected from the roof hits the wave vertically from the top, as seen in Figure $6 \mathrm{c}$. With an excitation frequency close to the first fundamental frequency, there is a single travelling wave as expected. The presence of tank-roof impact makes it difficult to compare the theory directly with the resulting sloshing regimes, since the additional energy dissipation from the impact is not accounted for in the formulas for natural frequencies. In case 3 , a wave is found that travels back and forth with greater speed. The impact against the wall and roof is more severe and creates a slap noise. The wave builds up and has a maximum amplitude few centimetre from the wall, as shown in Figure 6e. The wave is about to break just before it hits the wall. By increasing the frequency slightly, to $f / f_{1,0}=1.44$, the sloshing regimes change dramatically in case 4 as seen in Figure $6 \mathrm{~g}$, where the amplitude is smaller.

The comparison between experiments and simulations is based on the free surface elevation at a single point, at the location of the hinge which is $0.408 \mathrm{~m}$ from the left wall of

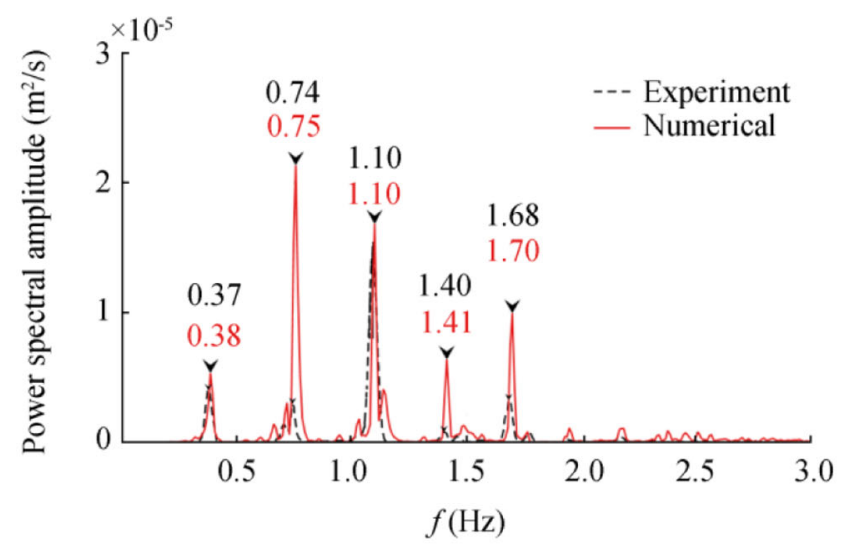

(a) Natural frequencies $f_{1,0}$ to $f_{5,0}$ in time window $40-80 \mathrm{~s}$

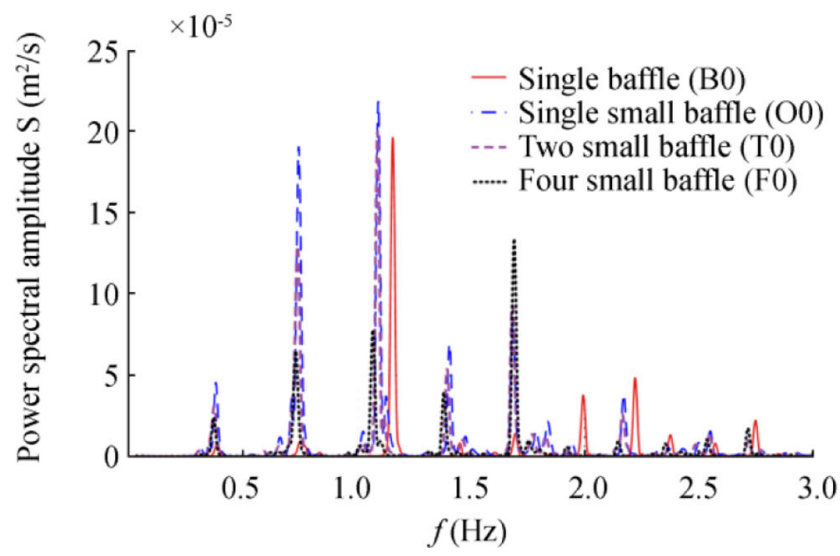

(b) Free sloshing with baffles- simulations B0, S0, T0 and F0

Figure 5 FFT of the free surface elevations under free sloshing without baffles and free sloshing with baffles

the sloshing tank. The results from cases 1-4 are plotted in Figure $7 \mathrm{a}-\mathrm{d}$, respectively, and a good agreement is seen. The single travelling wave is predicted well with some discrepancies due to small-scale ripples on the wave in the experiments. In cases $1-3$, the free surface elevations clearly show the single travelling wave with two peaks within each cycle. In case 3 , it can be seen that the two peaks have almost the same amplitude because the amplitude of the travelling wave differs only slightly as it travels back and forth between the two side walls during sloshing. The primary mode is dominating, but in case 4 , higher modes become visible, where secondary and tertiary crests appear on the free surface as seen in Figure $7 \mathrm{~d}$. Some spurious recordings are observed in the measurements of the free surface in case 4 , which are seen to be clear outliers and are ignored. The numerical results for case 4 (N6) do not show such a discrepancy.

The power spectrum of the free surface amplitude in each case of forced sloshing is given in Figure 8a-d. In all the cases, it is seen that the peaks occur at the excitation frequency in the case and at higher harmonics of the excitation frequency. In 
Figure 6 Sloshing in the tank for experiments with different excitation frequencies

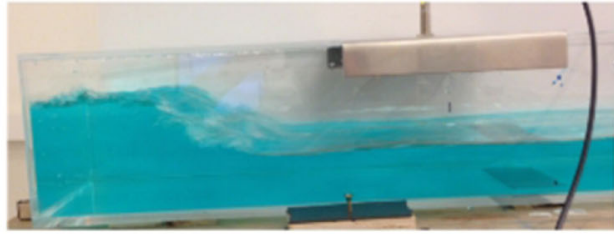

(a) Case $1\left(f / f_{1,0}=0.79\right)$

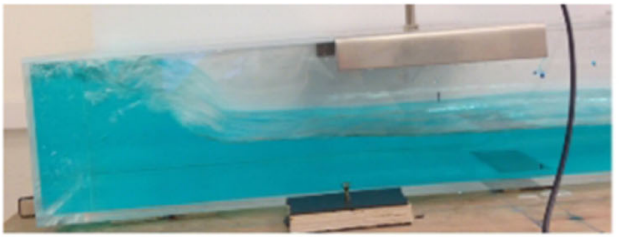

(c) Case $2\left(f / f_{1,0}=1.00\right)$

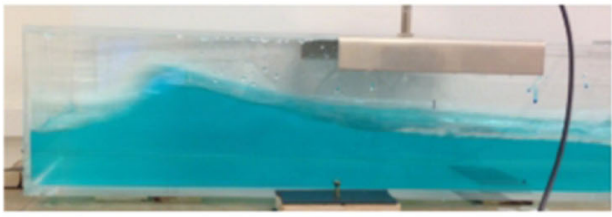

(e) Case $3\left(f / f_{1,0}=1.40\right)$

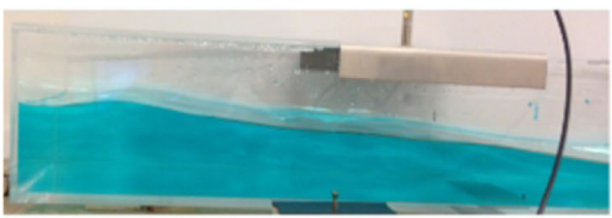

(g) Case $4\left(f / f_{1,0}=1.44\right)$

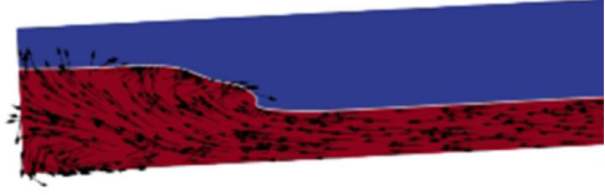

(b) Case N2 $\left(f / f_{1,0}=0.79\right)$

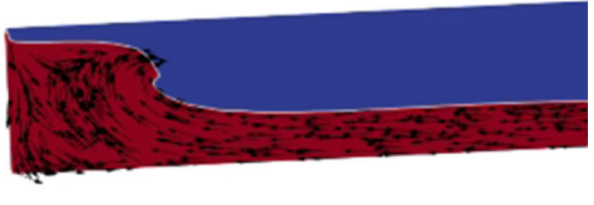

(d) Case N3 $\left(f / f_{1,0}=1.00\right)$

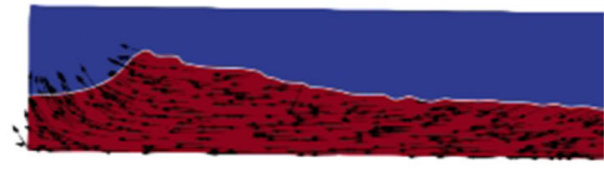

(f) Case N5 $\left(f / f_{1,0}=1.40\right)$

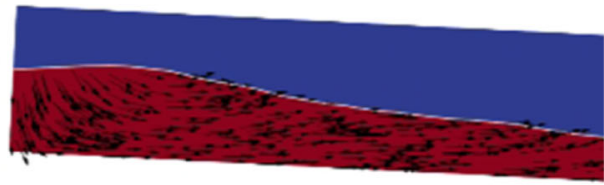

(h) Case N6 (f/f $\left.f_{1,0}=1.44\right)$ case 1, the highest peak is seen at the excitation frequency, which is below the fundamental frequency, and smaller spectral contributions are seen for the higher harmonics of the excitation frequency in Figure 8(a). In case 2, the excitation frequency, $f$, is close to $f_{1,0}$. The dominant frequency is seen to be twice the excitation frequency, indicating that the travelling wave due to sloshing does not undergo significant changes during its motion between the end walls and it registered twice as described in 4.2. It is also noticed that harmonics of the excitation frequency are close to the higher mode natural frequencies. This is because in shallow water, the harmonics of the first mode natural frequency, $n \cdot f_{1,0}$, are close to the natural frequencies of higher modes (see Table 1). This is referred to as commensurate spectrum (Faltinsen and Timokha 2009). The results for case 3 are similar to that seen in case 2, but the spectral amplitudes are lower due to energy dissipation from tank-roof impacts during sloshing. The difference between cases 3 and 4 is significant, and due to the presence of higher mode waves, accurate prediction of the waves is more dependent on the realistic representation of the dissipation of energy due to the deformation of the free surface. From the power spectrum in case 4 in Figure $8 d$, it can be noted that the excitation frequency and its harmonics are accurately predicted. The results indicate that the modes excited by the forcing are dominating in this case. The frequencies are accurately predicted by the simulations in all the cases, and the differences in the spectral amplitudes are due to the FFT procedure. This indicates that the numerical model is able to replicate the sloshing dynamics with acceptable accuracy, even in cases with higher harmonics and complex free surface deformations resulting in significant energy dissipation (e.g. case 4). Finally, it is noted that cases 3 and 4 have $2 f$ close to $f_{3,0}$ and the difference between the two cases is significant. This is due to a transition in the sloshing modes due to the presence of a resonance zone and is further investigated in the next section.

\subsection{Investigation of the Transition Region Between Cases 3 and 4}

Studies of sloshing in shallow water depths by Faltinsen and Timokha (2009) mention different wave systems at shallow depths with sway excitation, but with reference to studies 


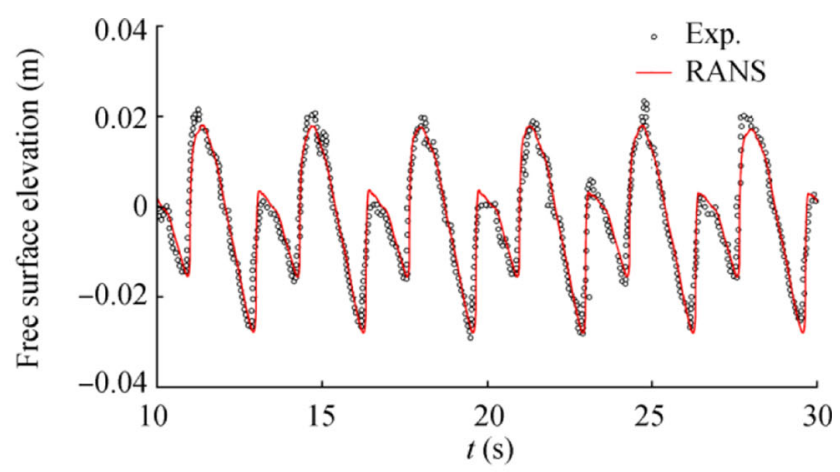

(a) Case $1\left(f / f_{1,0}=0.79\right)$

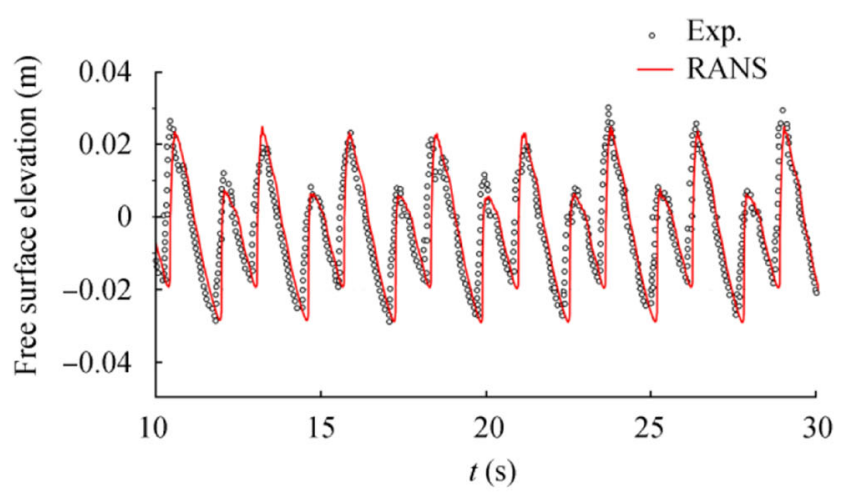

(b) Case $2\left(f / f_{1,0}=1.00\right)$

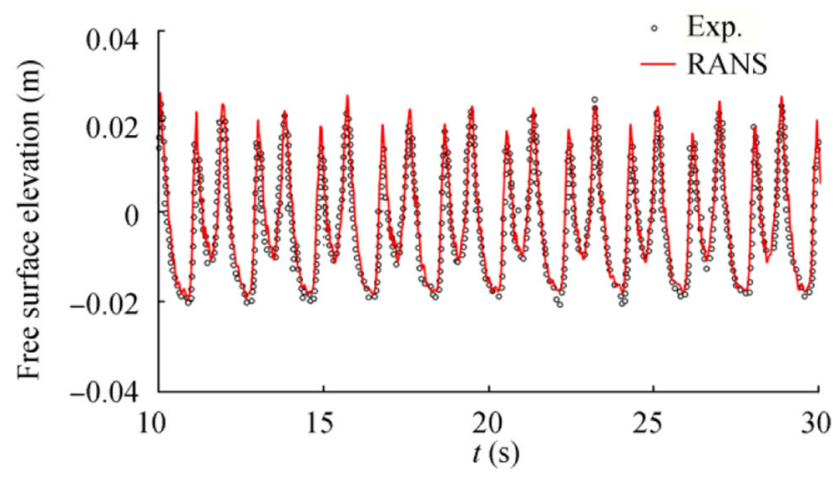

(c) Case $3\left(f / f_{1,0}=1.40\right)$

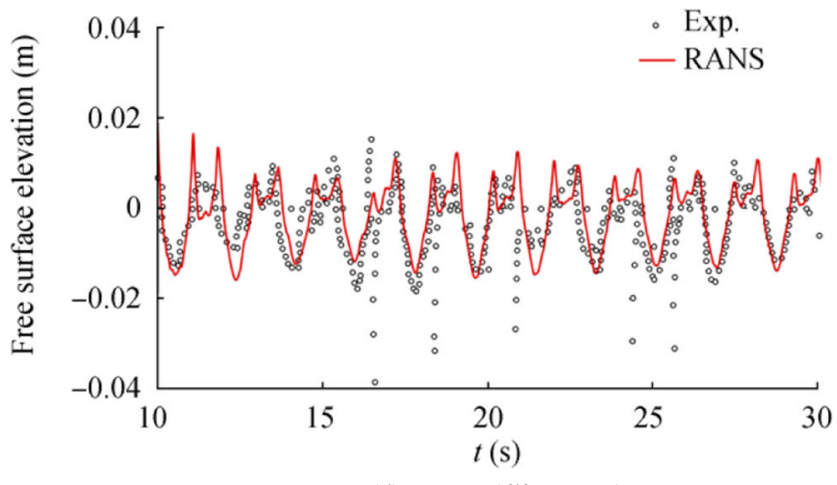

(d) Case $4\left(f / f_{1,0}=1.44\right)$

Figure 7 Comparison of experimental data and numerical results for free surface elevation in the tank under forced sloshing performed by Verhagen and Van Wijngaarden (1965). There is little information about the transition between these observed sloshing regimes at the upper limit of the resonance zone. It seems that the transition might be related to the proximity of $2 f$ to the third natural frequency. The third mode natural frequency is calculated to be $f_{3,0}=1.10 \mathrm{~Hz}$, but maybe close to $1.09 \mathrm{~Hz}$ according to the free sloshing test. The half of the calculated natural frequency $f_{3,0}=1.10 \mathrm{~Hz}$ is the same as the excitation frequency in case 4 , while the half of the observed $f_{3,0}=1.09 \mathrm{~Hz}$ gives $f=0.545 \mathrm{~Hz}$. Additional simulations with both $f=0.540 \mathrm{~Hz}$ and $f=0.545 \mathrm{~Hz}$ are performed and compared to the results for $f=0.550 \mathrm{~Hz}$ (case 4) in Figure 9. The results show the transition just after a few cycles when the excitation frequency is exactly equal to $f=1.09 / 2=$ $0.545 \mathrm{~Hz}$. As shown previously in Figure 8d, the amplitude in case 4 reaches a steady value at around $10 \mathrm{~s}$, while with the frequency $f=0.545 \mathrm{~Hz}$, the same amplitude as in case 4 is reached after approximately $16 \mathrm{~s}$. With $f=0.540 \mathrm{~Hz}$, the same amplitude is reached only after $50 \mathrm{~s}$.

\subsection{Effect of Filling Ratio and Roll Amplitude on Sloshing}

In the previous section, a resonance zone is present at a frequency equal to $f / f_{1,0}=1.40$ for $h / L=0.06$, and an amplitude of $3.0^{\circ}$. Previous investigations by Armenio and La Rocca (1996) with a similar filling ratio of $h / L=0.05$ and excitation frequency of $f / f_{1,0}=1.497 \mathrm{~Hz}$ showed a linear variation of the wave height below and above $3.0^{\circ}$, but with different slopes. As the excitation frequency in their case is higher than the frequency found for the transition case presented above, the difference in slope is most likely due to the transition in the sloshing regime. In this section, the study is extended through simulations to study the upper limit of the resonance region for different roll amplitudes and filling depths. Roll amplitudes of $1.7^{\circ}, 3.0^{\circ}$ and $6.0^{\circ}$ are considered. Simulations with a higher filling ratio of $h / L=0.10$ are carried out as listed in Table 2 . The numerical setup is the same as that illustrated in Figure 1a and the calculated fundamental frequency is $f_{1,0}=0.487 \mathrm{~Hz}$.

The results for the variation of the maximum free surface elevation ( $\eta \max$ ) in the middle of the tank for three different roll amplitudes, $1.7^{\circ}, 3.0^{\circ}$ and $6.0^{\circ}$, for eight different excitation frequencies and filling ratio $h / L=0.06$ are presented in Figure 10a. Previous results (Armenio and La Rocca 1996) showed a linear variation of the free surface elevation with a roll amplitude of $1.7^{\circ}$ within the frequency range $f=0.48$ $0.80 \mathrm{~Hz}$. The present results agree with those findings as seen from the linear increase in the normalised maximum free surface elevation $\left(\eta / \eta_{\text {max }}\right)$ until $f / f_{1,0}=1.32$, where the maximum is reached. It is seen from Figure 10a that a further increase in the excitation frequency to $f / f_{1,0}=1.40$ results in an immediate decrease in the maximum free surface elevation in the tank, and an extended region of resonance zone is not seen. An 


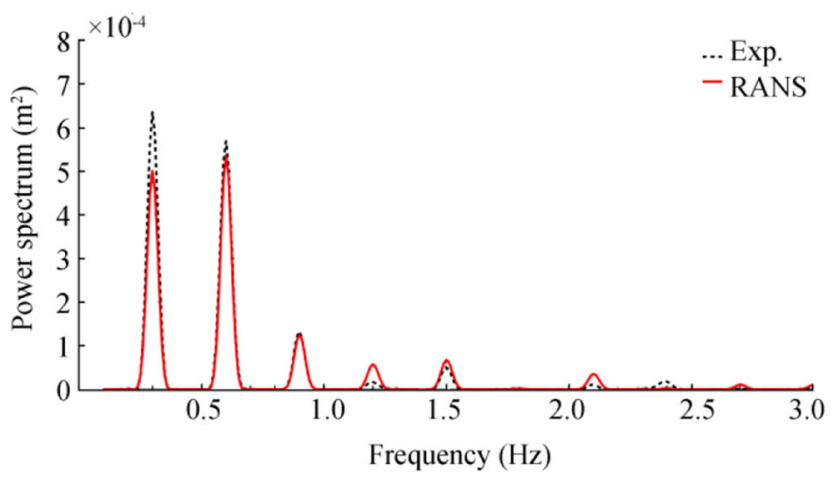

(a) Case $1\left(f / f_{1,0}=0.79\right)$

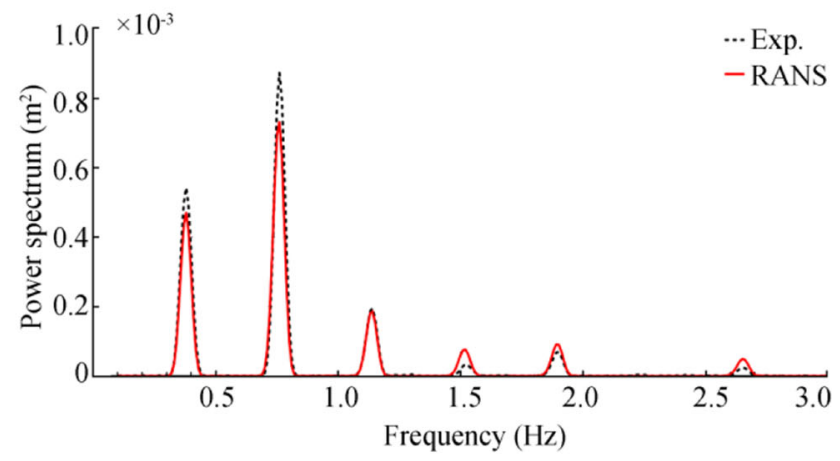

(b) Case $2\left(f / f_{1,0}=1.00\right)$

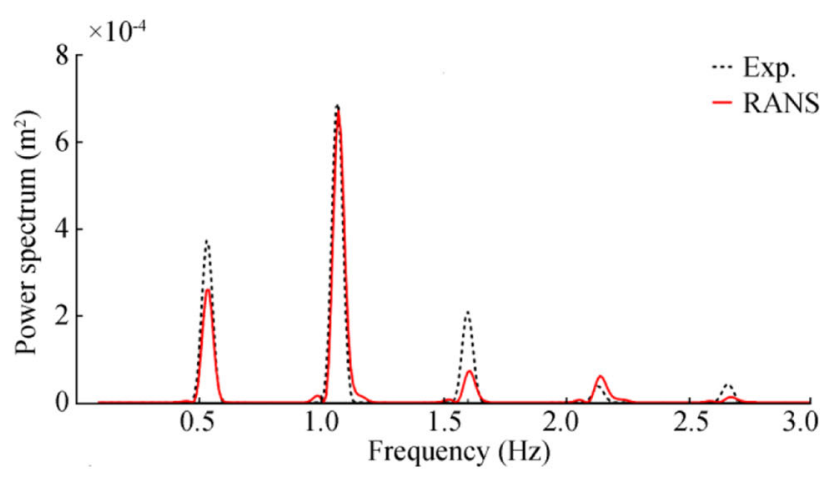

(c) Case $3\left(f / f_{1,0}=1.40\right)$

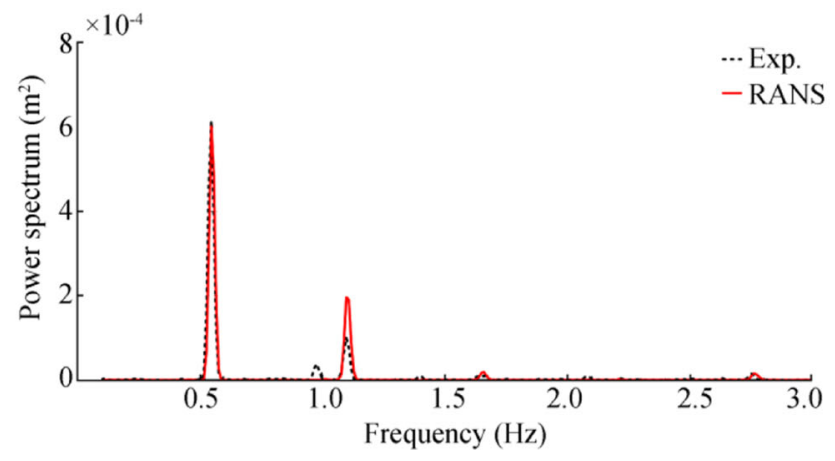

(d) Case $4\left(f / f_{1,0}=1.44\right)$

Figure 8 Power spectrum of the free surface elevation under forced sloshing at different frequencies

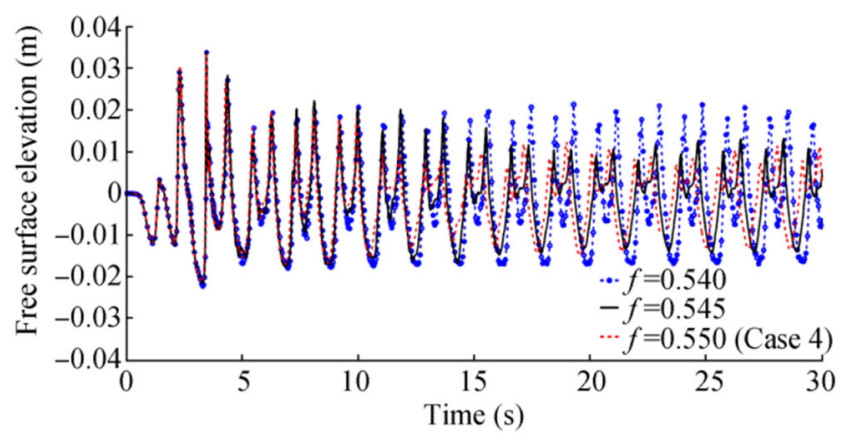

Figure 9 Transition in the upper limit of the resonance zone

additional simulation with $f=0.515 \mathrm{~Hz}$ with resulting $f / f_{1,0}=$ 1.36 is carried out to confirm this in Figure 10a. The result from the previous section regarding the region of resonance between $f / f_{1,0}=1.40$ and 1.43 is seen in the figure for a roll amplitude of 3 . On increasing the roll amplitude to $6^{\circ}$, the maximum free surface elevation is calculated for $f / f_{1,0}=$ 1.40 , and the upper limit of the resonance zone is seen to be around $f / f_{1,0}=1.50$. According to Eq. (4), the theoretical upper limit for the upper limit of the resonance zone is $f / f_{1,0}=1.36$, 1.47 and 1.67 for roll amplitudes of $1.7^{\circ}, 3.0^{\circ}$ and $6.0^{\circ}$, respectively. The results show that while Eq. (4) predicts a wide range for resonance and is considered poor for predictions for $h / L>0.04$, the upper limit of the resonance zone for $h / L=0.06$

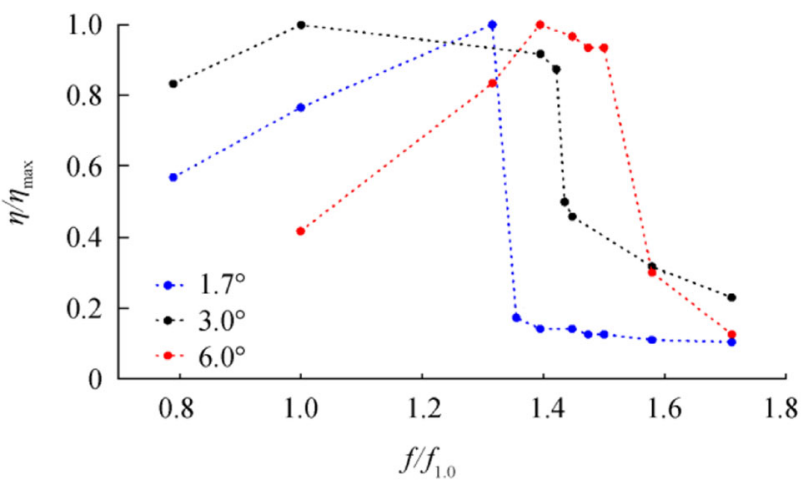

(a) Roll amplitudes $1.70^{\circ}, 3.0^{\circ}$, and $6.0^{\circ}$ with filling ratio $h / L=0.06$

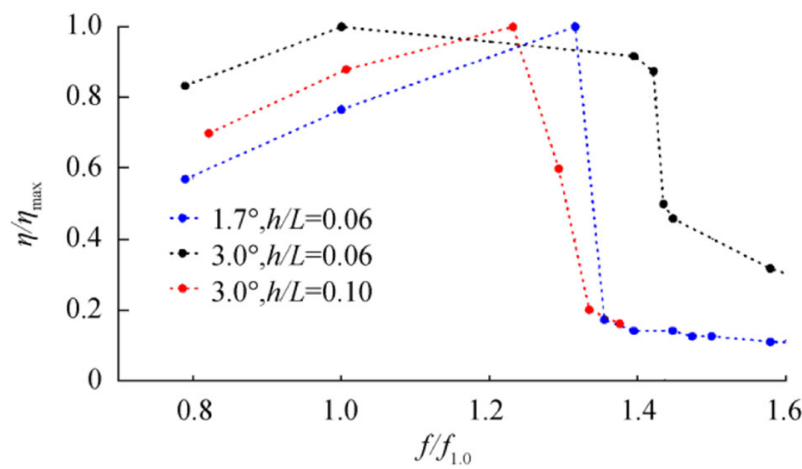

(b) Filling ratios $h / L=0.06$ and $h / L=0.10$ for roll amplitudes $1.70^{\circ}$ and $3.0^{\circ}$

Figure 10 Variation of the free surface elevation for different excitation frequencies with higher roll amplitude and filling ratio 
is predicted within reasonable limits when the roll amplitude is small.

The variation of the maximum free surface elevation for filling ratios $h / L=0.06$ and $h / L=0.10$ and roll amplitude $3^{\circ}$ along with $h / L=0.06$ and roll amplitude $1.7^{\circ}$ are presented in Figure 10b. The results show that the upper limit of the resonance zone for a higher filling ratio $h / L=0.10$ is about $f / f_{1,0}=$ 1.23 , while the maximum free surface elevation for a lower filling ratio $h / L=0.06$ is seen for $f / f_{1,0}=1.00$ for the same roll amplitude of $3^{\circ}$. Further, it is also observed that for the lower filling ratio $h / L=0.06$, the maximum free surface elevation occurs at $f / f_{1,0}=1.32$ which is higher than that seen for $h / L=$ 0.10 . The theoretical upper limit of the resonance zone for $h / L$ $=0.10$ and roll amplitude $3^{\circ}$ is $f / f_{1,0}=1.38$. The presented results show that with an increase in the filling ratio for a given roll amplitude, the resonance moves to a higher $f / f_{1,0}$. Also the variation of the maximum free surface elevation at a higher filling ratio and roll amplitude is similar to the variation at a lower filling ratio and lower roll amplitude.

\subsection{Forced Sloshing with a Single Baffle}

The effect of baffles on the sloshing in the tank is investigated with a single baffle inside the tank, $0.3 \mathrm{~m}$ from the tank wall on the right. The free surface measurements are compared to the simulations for two different excitation frequencies $0.23 \mathrm{~Hz}$ and $0.30 \mathrm{~Hz}$ in experimental cases 6 and 7, respectively, which correspond to simulations B1 and B2, respectively. The measured and calculated free surface elevations in the tank are presented in Figure $11 \mathrm{a}$ and $\mathrm{b}$, respectively. The baffle is tall enough to be surface piercing when the tank is at one extreme end of its rotation. The amplitude of the largest wave in case 7 is slightly under-predicted, but in general the discrepancy is small.

Snapshots from the simulations and experiments in case 7 are in Figure $12 \mathrm{a}-\mathrm{h}$. In Figure $12 \mathrm{a}$, the low pressure to the right of the baffle creates suction and a wave rolls up in the opposite direction. The markers denoting the direction of the flow in Figure 12b clearly show the submerged flow under the surface roller to the right of the baffle. In Figure 12c, a wave is running from left to right towards the baffle. The flow markers in Figure 12d also represent the surface flow towards the baffle from left to right along with a submerged flow going right to left away from the baffle. The wave hits the baffle in Figure $12 \mathrm{e}$ with a slap noise in the experiments. The airwater mixture and bubble formation due this splashing is not represented by the model due to the nature of the level set method in these simulations, but the deformation of the free surface in the vicinity of the baffle is reasonably represented in Figure 12f. The overtopping of the baffle and the associated splashing is seen in Figure $12 \mathrm{~g}$. In the simulations, the free surface apart from the splash is represented well by the model. The discrepancy in the presented results for the free surface

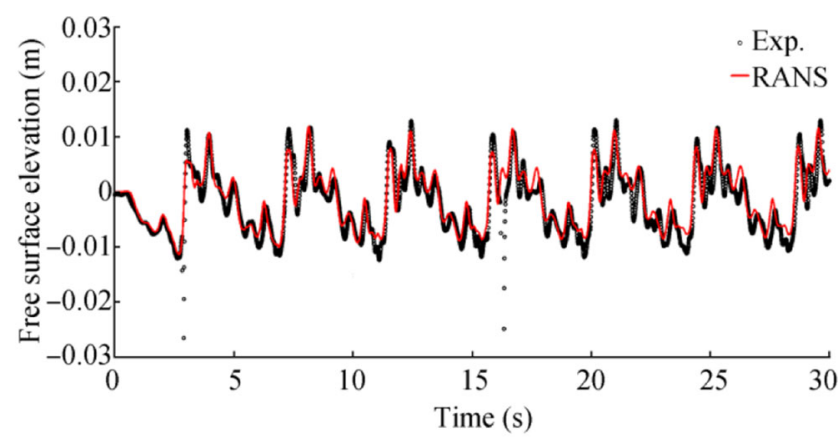

(a) Case 6 (expt) and B1 (num) for $f / f_{1,0}=0.61$

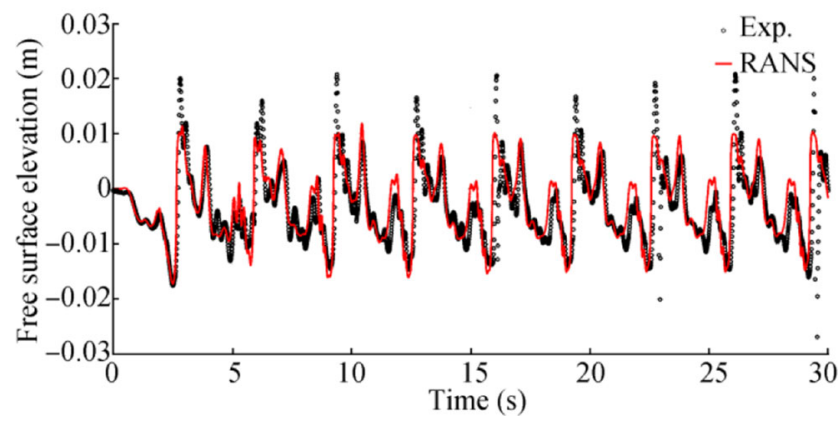

(b) Case 7 (expt) and B2 (num) for $f / f_{1,0}=0.79$

Figure 11 Comparison of the experimental and numerical results for the free surface elevation with a single baffle

elevation in Figure $11 \mathrm{~b}$ can be accounted for from the splashing process that results in a higher free surface recording in the experiments which is absent in the numerical simulations.

Further the variation of the maximum free surface elevation for in the tank under different excitation frequencies and roll amplitude $3^{\circ}$ is investigated with simulations listed in Table 3. The results are presented in Figure 13 variation along with the variation of the free surface in the simulations without baffles. The following effect due to the presence of the baffle in the tank is seen from these results. The maximum free surface elevation is calculated for $f / f_{1,0}$ $=1.58$ with an absolute value of $0.021 \mathrm{~m}$. This is only slightly lower than the maximum free surface elevation seen in the simulations without baffles, that is, $0.023 \mathrm{~m}$ in simulation B2 with $f / f_{1,0}=1.00$. The lowest free surface elevation for sloshing with baffles is calculated for $f / f_{1,0}=$ 1.00. The free surface elevations are never less than 0.8 times the maximum for the range of frequencies simulated. This is in contrast to the simulations without baffles where the free surface elevations are strongly reduced after $f / f_{1,0}=$ 1.58. These results indicate that while the presence of the baffle results in a reduction of the free surface elevation under sloshing, certain conditions can result in higher free surface elevations compared to sloshing without baffles. 
Figure 12 Segment of the physical (case 7) and numerical sloshing tanks (B2) with $f=0.3 \mathrm{~Hz}$ and a single baffle

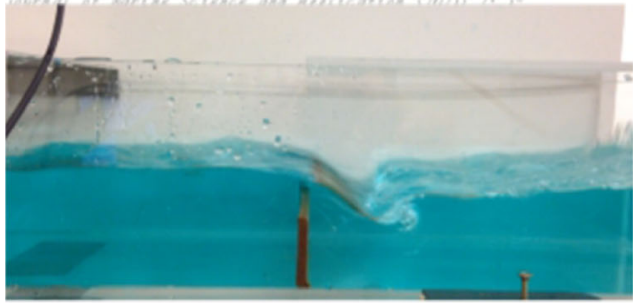

(a) Experiment $t=3.86 \mathrm{~s}$

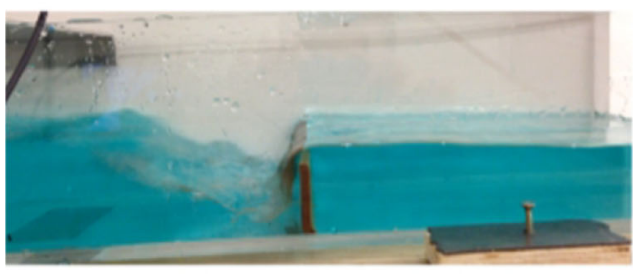

(c) Experiment $t=6.18 \mathrm{~s}$

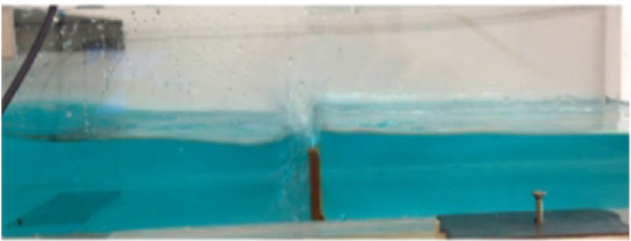

(e) Experiment $t=6.38 \mathrm{~s}$

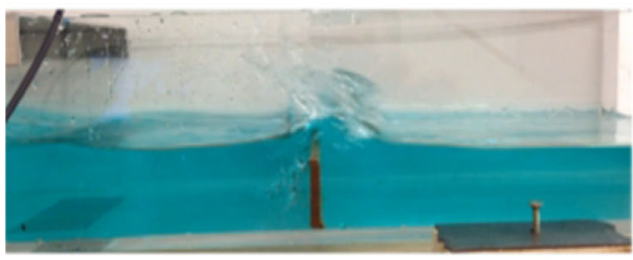

(g) Experiment $t=6.50 \mathrm{~s}$

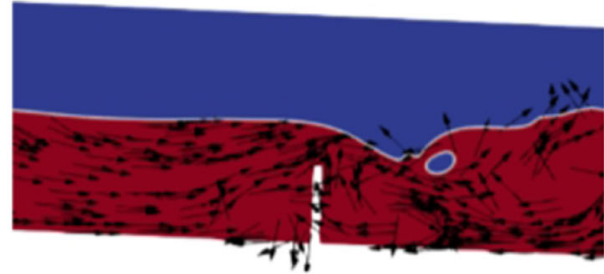

(b) Simulation $t=3.86 \mathrm{~s}$

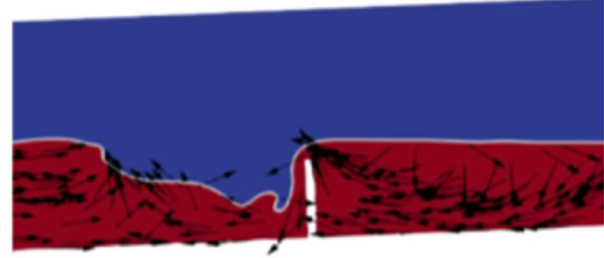

(d) Simulation $t=6.18 \mathrm{~s}$

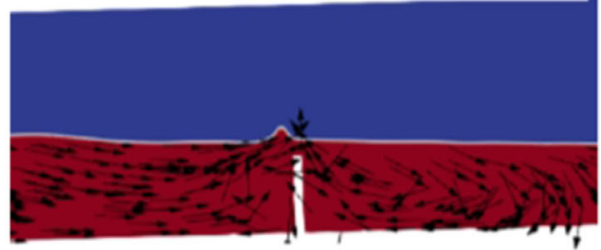

(f) Simulation $t=6.38 \mathrm{~s}$

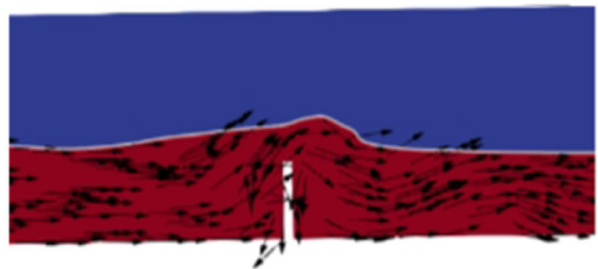

(h) Simulation $t=6.50 \mathrm{~s}$

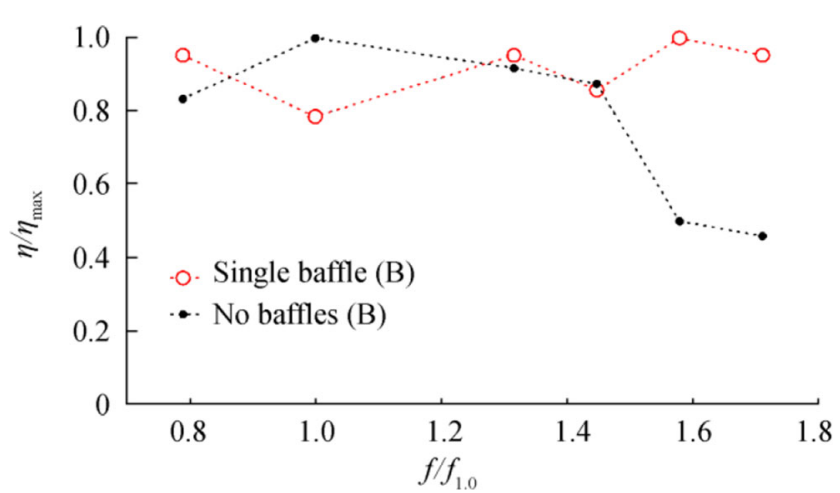

Figure 13 Variation of the free surface elevation for different excitation frequencies for sloshing with a single tall baffle

\subsection{Forced Sloshing with Small Baffles}

In this section, the results from simulations listed in Table 4 with one, two and four short baffles are presented. The baffles used in these simulations are referred to as small baffles as they do not pierce the free surface under sloshing and the baffle to mean water depth ratio is 0.25 . The variation of the free surface elevation in the tank over the excitation frequencies simulated for the three different arrangements of short baffles is presented in Figure 14.

It is clear from this figure that the presence of the baffles has a strong dissipative effect on the free surface in the tank at all frequencies except $f / f_{1,0}=1.32$. It is also seen that the reduction of the free surface elevation under sloshing is similar for all the simulated arrangements of the baffles. The maximum free surface elevation in all three cases is seen at $f / f_{1,0}=$ 1.32 , and the minimum is seen at $f / f_{1,0}=1.58$ with $0.19 \eta_{\max }$, 


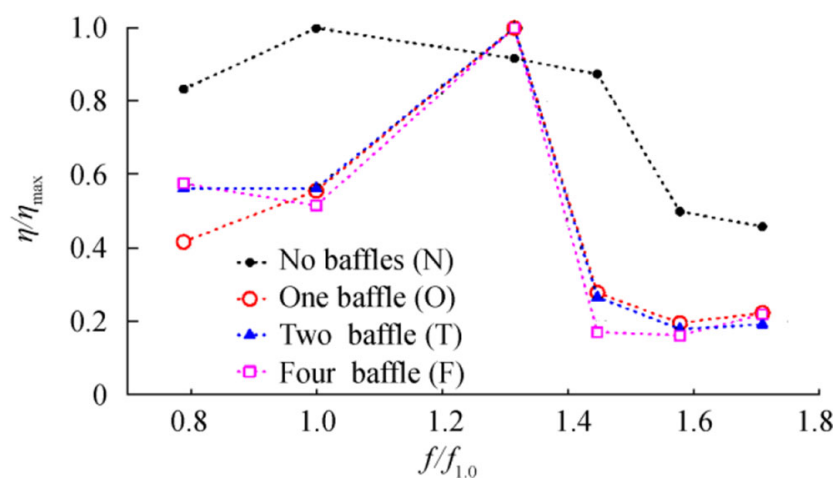

Figure 14 Variation of the free surface elevation for different excitation frequencies for sloshing with short baffles

$0.18 \eta_{\max }$ and $0.16 \eta_{\max }$ for the simulations with one, two and four baffles, respectively. The absolute values of the maximum free surface elevation $\left(\eta_{\max }\right)$ in the simulations with one, two and four short baffles are quite similar and are calculated to be $0.036,0.032$ and $0.033 \mathrm{~m}$, respectively. From this information and the results presented in Figure 14, it can be deduced that the configuration with two and four short baffles provide reduced sloshing motion the most. The exception is at $f / f_{1,0}=0.8$, where the configuration with one short baffle results in the lowest free surface elevation.

The results in Figure 14 can be contrasted with those seen in Figure 13 for a single tall baffle. The major difference is that with a single tall baffle, the free surface elevations calculated for the frequency range under study are never under $0.80 \eta_{\max }$, whereas there is significant reduction of the free surface elevation for most excitation frequencies used in the study. A similarity that is noticed is that the maximum free surface elevation is calculated at $f / f_{1,0}=1.32$ for the single tall baffle and for the short baffle configurations.

The free surface elevations under sloshing for the simulation with a single tall baffle (B3) and two short baffles (T3) for excitation frequency $f / f_{1,0}=1.00$ are presented in Figure 15 along with markers indicating the direction of the flow. The free surface elevations calculated close to the middle of the tank at $0.408 \mathrm{~m}$ from the left wall and close to the left wall at $0.05 \mathrm{~m}$ from the wall for the two cases presented above are shown in Figure 16. From the time series of the free surface elevations at the two locations in the two simulations and the motion of the free surface presented in Figure 15, the following observations can be made.

The free surface in the tank at $t=11.82 \mathrm{~s}$, when the tank returns to its neutral position after rotation to the right in the case with two short baffles presented in Figure 15a, the flow is seen to be meeting from both directions in the region around the two baffles. Due to this, the free surface near the centre of the tank is characterised by the presence of a surface roller and wave breaking in the simulation with four short baffles as seen from Figure 15a and supported by the near vertical slope of the free surface elevation around the same time in Figure 16a. In contrast, at the same simulation time, the formation of a strong surface roller is absent for the simulation with the single tall baffle as seen from Figures 15b and 16a, where the free surface does not have a steep gradient. The fluid flow on the left side of the baffle is seen to be mostly directed towards the left, whereas two recirculation zones are seen in the fluid on the right side of the baffle.

At $12.44 \mathrm{~s}$ in the simulations, the tank is at its maximum rotational position to the left. In the simulation with two short baffles in Figure 15c, the flow is mostly directed right to left in the entire tank. There is flow overtopping the baffle and run up along the left side wall is seen. The flow features are seen to be the similar for the simulation with a single tall baffle in Figure 15d. Figure 16a shows that when the tank is close to its extreme position, the free surface elevation near the centre is close to the mean water level in the simulation with four short baffles, whereas for the simulation with a single tall baffle, the free surface elevation is closer to one of the local minima. The run up along the walls is seen to be slightly higher for case with two short baffles compared to the case with a single tall baffle as seen in Figure 16b.

The tank returns to its neutral position at $t=13.22 \mathrm{~s}$ in the simulations. In the simulation with two short baffles, the flow is directed left to right on the left side of the right baffle in Figure $15 \mathrm{e}$. There is run down from the left wall, and overtopping of the left baffle. The region to the right of the right baffle has flow going right to left. The two opposing flows result in a surface roller in the region between the right baffle and the right wall of the tank. The time series in Figure 16a indicates that the roller formed at this instant has a lower height than that formed earlier at $t=11.82 \mathrm{~s}$. At the same simulation time, Figure $15 \mathrm{f}$ shows that the flow is directed left to right to the left of the baffle, and two weak recirculation zones are seen to the right of the baffle for the simulation with the single tall baffle. Air entrainment in the region between the baffle and the impacting fluid travelling from the left is seen. The surface roller resulting in this impact is formed at around $t=12.8 \mathrm{~s}$ according to the steep gradient in the free surface seen in Figure 16a. During the time the tank is close to its neutral position, the slope of the free surface elevations near the wall is quite gentle in both simulations as seen in Figure 16b.

The tank is at its maximum rotational position to the right at $t=13.82 \mathrm{~s}$ in the simulations. In the simulation with two short baffles presented in Figure $15 \mathrm{~g}$, the flow is mostly directed left to right, and a high run up along the right wall is seen. The flow scenario is similar in the simulation with the single tall baffle in Figure 15h, but in addition, a surface roller is seen to the right of the baffle moving opposite to the principal flow direction. When the tank is at its extreme position on either side in the process of sloshing, the highest run up along the walls is on the same side as the maximum rotation for both simulations. 
Figure 15 Free surface in the tank with velocity contours for case B3 with one tall baffle and case T3 with two short baffles

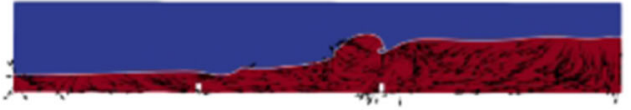

(a) $\mathrm{B} 3, t=11.82 \mathrm{~s}$

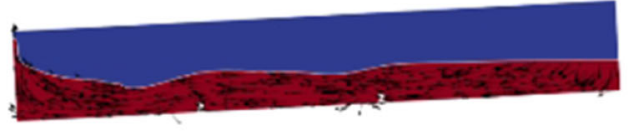

(c). B3, $t=12.44 \mathrm{~s}$

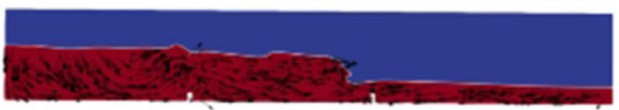

(e). B3, $t=13.22 \mathrm{~s}$

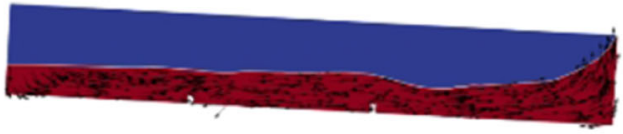

(g) B3, $t=13.82 \mathrm{~s}$

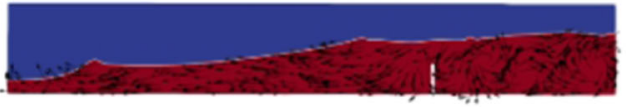

(b) $\mathrm{T} 3, t=11.82 \mathrm{~s}$

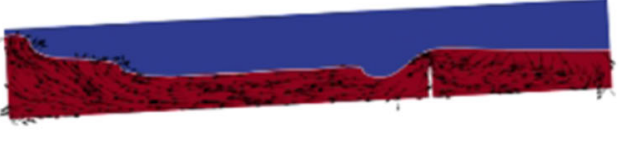

(d) $\mathrm{T} 3, t=12.44 \mathrm{~s}$

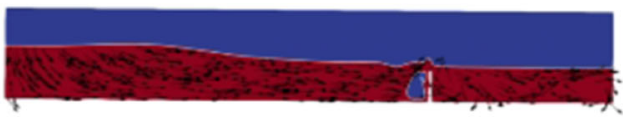

(f) $\mathrm{T} 3, t=13.22 \mathrm{~s}$

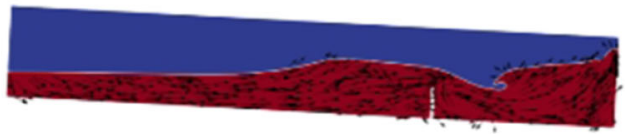

(h) T3, $t=13.82 \mathrm{~s}$
Figure 16 shows that the period of the free surface variation calculated in the middle of the tank is close to two times the period of the variation near the wall for the simulation with two short baffles. In addition, the free surface elevation near the centre of the tank is dominated by one wave crest for the simulation with two short baffles, whereas for the case with

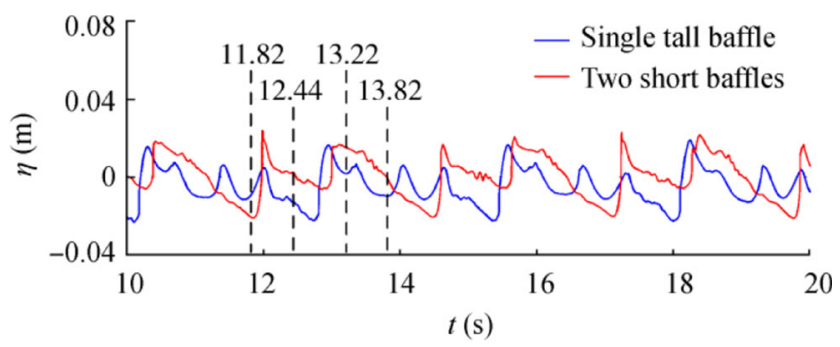

(a) Close to the centre of the tank, at $x=0.408 \mathrm{~m}$

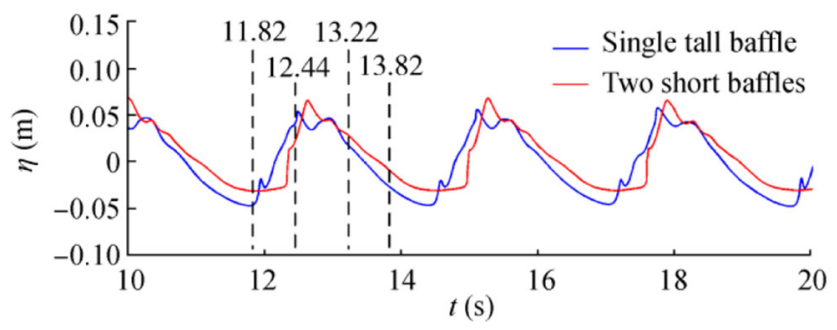

(b) Close to the left wall, at $x=0.05 \mathrm{~m}$

Figure 16 Time series of the free surface elevations calculated in simulations B3 and T3 with a single tall baffle and two short baffles respectively the single tall baffle, up to four local wave crests are seen before the minimum free surface elevation is reached in Figure 16a. On the other hand, Figure 16b shows that the variation in the free surface elevation near the wall is similar in both simulations.

\section{Conclusions}

The free surface elevations from sloshing experiments and RANS simulations are compared for several cases with shallow filling depths in a rectangular tank. The experiments are conducted at the lab facility at NTNU Ålesund.

The RANS simulations are performed using the opensource CFD solver REEF3D. Cases with one specific filling for a clean tank for validation and a slightly increased filling for a tank with a single baffle are investigated in the experiments. The roll excitation frequencies of the tank are in proximity to the first mode natural frequency. The numerical mod$\mathrm{el}$ is validated by comparing the time series of the measured free surface elevations in the experiments and the Fourier transformation of the time series to the corresponding numerical results. The numerical model is able to replicate the experimental observations well. The splashing of the liquid during its impact with a single baffle is not captured due to the nature of the level set method, while the form of the main fluid body is well accounted for. A sudden reduction in the sloshing amplitude is observed in the experiments when the excitation 
frequency is increased from $f / f_{1,0}=1.40$ to 1.44 , and numerical simulations are used to investigate the transition region.

Further, the extent of the resonance region under different roll amplitudes and filling ratios are investigated. It is found that the resonance zone spans a wide frequency range as the roll amplitude is increased for the filling ratio investigated in the study. It is also found that the extent of the resonance zone is reduced for a higher filling ratio for the same roll amplitude.

The numerical model is then used to study the sloshing regime with baffles. Free sloshing simulations demonstrated that the natural frequencies in the tank are close to the same as those found in the configuration without baffles. It is then found that with the single baffle configuration used in the study with baffle to water depth ratio 0.72 , the free surface elevation is not reduced below 0.80 of the maximum free surface elevation over different excitation frequencies. The occurrence of the maximum free surface elevation in the tank is moved from around the first mode to a higher mode. Further, studies are conducted with one, two and four short baffles, with a baffle to water depth ratio of 0.25 . The results show that the variation of the maximum free surface elevation over the range of excitation frequencies investigated in the study is similar for all three configurations. The same is seen for the flow features in the sloshing tank. The maximum free surface elevation at the centre of the tank is seen for $f / f_{1,0}=$ 1.32 , whereas the minimum is seen for $f / f_{1,0}=1.58$. The run up on the sidewalls is seen to be higher for the simulations with the short baffles compared to the simulations with the tall baffle. Energy dissipating surface rollers are formed twice per sloshing cycle for the short baffle compared to the tall baffle, and therefore, configuration with the short baffles are seen to reduce the sloshing amplitudes better than the configuration with the single tall baffle.

The numerical model is seen to replicate the sloshing at shallow filling depths quite well and offers interesting insights into the flow physics and design of baffles. Further studies can extend the knowledge about sloshing dynamics through the investigation of the optimal number, size and placement of baffles, lateral forces on the wall and the baffles and the excitation frequencies at which the maximum forces occur.

Funding Open access funding provided by NTNU Norwegian University of Science and Technology (incl St. Olavs Hospital Trondheim University Hospital).

Open Access This article is licensed under a Creative Commons Attribution 4.0 International License, which permits use, sharing, adaptation, distribution and reproduction in any medium or format, as long as you give appropriate credit to the original author(s) and the source, provide a link to the Creative Commons licence, and indicate if changes were made. The images or other third party material in this article are included in the article's Creative Commons licence, unless indicated otherwise in a credit line to the material. If material is not included in the article's Creative Commons licence and your intended use is not permitted by statutory regulation or exceeds the permitted use, you will need to obtain permission directly from the copyright holder. To view a copy of this licence, visit http://creativecommons.org/licenses/by/4.0/.

\section{References}

Ahmad N, Bihs H, Myrhaug D, Kamath A, Arntsen ØA (2018) Threedimensional numerical modelling of wave- induced scour around piles in a side-by-side arrangement. Coast Eng 138:132-151

Antuono M, Bouscasse B, Colagrossi A, Lugni C (2012) Twodimensional modal method for shallow-water sloshing in rectangular basins. J Fluid Mech 700:419-440

Armenio V, La Rocca M (1996) On the analysis of sloshing of water in rectangular containers: numerical study and experimental validation. Ocean Eng 23(8):705-739

Berthelsen PA, Faltinsen OM (2008) A local directional ghost cell approach for incompressible viscous flow problems with irregular boundaries. J Comput Phys 227(9):4354-4397

Bihs H, Kamath A (2017) A combined level set/ghost cell immersed boundary representation for floating body simulations. Int J Numer Methods Fluids 83(12):905-916

Bihs H, Kamath A, Alagan Chella M, Aggarwal A, Arntsen ØA (2016) A new level set numerical wave tank with improved density interpolation for complex wave hydrodynamics. Comput Fluids 140:191208

Durbin PA (2009) Limiters and wall treatments in applied turbulence modeling. Fluid Dynamics Research 41(1):012203

Faltinsen OM, Timokha AN (2002) Asymptotic modal approximation of nonlinear resonant sloshing in a rectangular tank with small fluid depth. J Fluid Mech 470:319-357

Faltinsen OM, Timokha AN (2009) Sloshing. Cambridge University Press

Faltinsen OM, Rognebakke OF, Lukovsky IA, Timokha AN (2000) Multidimensional modal analysis of nonlinear sloshing in a rectangular tank with finite water depth. J Fluid Mech 407:201-234

Grotle EL, Bihs H, Æsøy V (2017) Experimental and numerical investigation of sloshing under roll excitation at shallow liquid depths. Ocean Eng 138:73-85

Grotle EL, Bihs H, Æsøy V, Pedersen E (2018) Computational fluid dynamics simulations of nonlinear sloshing in a rotating rectangular tank using the level set method. Journal of Offshore Mechanics and Arctic Engineering 140(6):061806

Harten A (1983) High resolution schemes for hyperbolic conservation laws. J Comput Phys 49(3):357-393

Hossain M, Rodi W (1980) Mathematical modelling of vertical mixing in stratified channel flow. Proceedings of the 2nd Symposium on Stratified Flows. Trondheim:280-290

Ibrahim RA (2005) Liquid sloshing dynamics: theory and applications. Cambridge University Press

Ibrahim RA, Pilipchuk V, Ikeda T (2001) Recent advances in liquid sloshing dynamics. Appl Mech Rev 54(2):133-199

Jiang GS, Peng D (2000) Weighted ENO schemes for Hamilton-Jacobi equations. SIAM J Sci Comput 21:2126-2143

Jiang G, Shu C (1996) Efficient implementation of weighted ENO schemes. J Comput Phys 126(130):202-228

Jung J, Yoon H, Lee C, Shin S (2012) Effect of the vertical baffle height on the liquid sloshing in a three-dimensional rectangular tank. Ocean Eng 44:79-89

Kamath A, Bihs H, Arntsen ØA (2017) Study of water impact and entry of a free-falling wedge using computational fluid dynamics simulations. Journal of Offshore Mechanics and Arctic Engineering 139(3):031802 
Keulegan GH (1959) Energy dissipation in standing waves in rectangular basins. J Fluid Mech 6(1):33-50

Lu L, Jiang SC, Zhao M, Tang GQ (2015) Two-dimensional viscous numerical simulation of liquid sloshing in rectangular tank with/ without baffles and comparison with potential flow solutions. Ocean Eng 108:662-677

Menter FR (1994) Two-equation eddy-viscosity turbulence models for engineering applications. AIAA J 32(8):1598-1605

Miles JW (1967) Surface-wave damping in closed basins. Proceedings of the Royal Society of London A: Mathematical, Physical and Engineering Sciences 297(1451):459-475

Naot D, Rodi W (1982) Calculation of secondary currents in channel flow. J Hydraul Div 108(8):948-968

Ong MC, Kamath A, Bihs H, Afzal MS (2017) Numerical simulation of free-surface waves past two semi-submerged horizontal circular cylinders in tandem. Mar Struct 52:1-14

Osher S, Sethian JA (1988) Fronts propagating with curvature-dependent speed: algorithms based on Hamilton- Jacobi formulations. J Comput Phys 79(1):12-49
Peng D, Merriman B, Osher S, Zhao H, Kang M (1999) A PDE-based fast local level set method. J Comput Phys 155(2):410-438

Sussman M, Smereka P, Osher S (1994) A level set approach for computing solutions to incompressible two-phase flow. J Comput Phys 114(1):146-159

Van der Vorst HA (1992) Bi-CGSTAB: A fast and smoothly converging variant of $\mathrm{Bi}-\mathrm{CG}$ for the solution of nonsymmetric linear systems. SIAM J Sci Stat Comput 13(2):631-644

Verhagen J, Van Wijngaarden L (1965) Non-linear oscillations of fluid in a container. J Fluid Mech 22(4):737-751

Wilcox D (1994) Turbulence modeling for CFD. DCW Industries, Incorporated, La Canada, California, USA

Wu CH, Faltinsen OM, Chen BF (2012) Numerical study of sloshing liquid in tanks with baffles by time-independent finite difference and fictitious cell method. Comput Fluids 63:9-26

Zhao Y, Chen HC (2015) Numerical simulation of 3d sloshing flow in partially filled LNG tank using a coupled level-set and volume-offluid method. Ocean Eng 104:10-30 